Heinz Steinbring

\title{
Zur Entwicklung des Wahrscheinlichkeitsbegriffs Bernoullis Theorem aus didaktischer Sicht
}

\author{
In: Journal für Mathematik-Didaktik
}

Jg. 1, 1980, H. 3

S. 115-141 


\section{SONDERDRUCK \\ SONDERDRUCK}

\section{Ferdinand Schöningh}

Paderborn · München $\cdot$ Wien $\cdot$ Zürich 


\section{Heinz Steinbring}

\section{Zur Entwicklung des Wahrscheinlichkeitsbegriffs - Bernoullis Theorem aus didaktischer Sicht ${ }^{\star}$}

Summary: The teaching of probability, with special regard to the development of "stochastic thinking" requires a deeper consideration of pertinent applications. This is the only way to gain an appropriate understanding of the probability concept. Otherwise, however the theoretical character of this concept is indispensable within the context of applications. Each measurement of probability presupposes this concept itself. The paper dis- . cusses these difficulties by analyzing the historical development of Bernoulli's theorem: The passage from a primarily combinatorical to a more "statistical" concept of probability requires to take into account this concept's theoretical character. The main intentions of this paper focus on an epistemological analysis of Bernoulli's theorem; this is followed by some more general didactical conclusions, which will require further investigation for concrete problems in curriculum construction.

\section{Einleitung}

Es gibt wohl kaum einen mathematischen Begriff, der eine ähnliche Vielfalt verschiedenartiger Definitionen und gegensätzlicher Interpretationen aufweist, wie der Begriff der wahrscheinlichkeit: Da sind z.B. die Gleichwahrscheinlichkeit und die klassische Laplacesche Definition von Wahrscheinlichkeit, die Häufigkeitstheorie von Richard von Mises, die logische Wahrscheinlichkeit (etwa von Keynes) und schließlich die axiomatische Begründung der Wahrscheinlichkeit durch Kolmogoroff. Berücksichtigt man zudem die schon lang andauernde heftige Diskussion um die subjektive und objektive wahrscheinlichkeitsauffassung, so kann man insgesamt kendall nur zustimmen, der sagt: "Few branches of scientific method have been subject to so much difference of opinion as the theory of probability." (Kenda11, 1949, S. 101) Dies alles macht den Wahrscheinlichkeitsbegriff zwar zu einem sehr interessanten, aber gleichzeitig wohl auch zu einem der problematischsten mathematischen Begriffe für die Schule überhaupt.

* Für kritische Anmerkungen und Verbesserungsvorschläge möchte ich mich bei R. Biehler, H.N. Jahnke, Chr. Keitel, M. Otte und R. Sträßer bedanken. 
Andererseits wird sehr häufig gerade die Wahrscheinlichkeitstheorie als Paradebeispiel der Anwendbarkeit von Mathematik in anderen Wissenschaften, wie etwa der Physik, Biologie, Soziologie etc. und auch in vielen praktischen Bereichen angeführt. Dieser zugleich grundlegende und vielseitige charakter des Wahrscheinlichkeitsbegriffs ist vielen Mathematikern und Didaktikern ein Grund dafür, die Wahrscheinlichkeitstheorie ins schulcurriculum einzubeziehen und "die schüler frühzeitig mit dem stochastischen Denken in Berührung zu bringen" (Dinges, 1978, s. 111).

Angesichts dieser Situation stellt sich das problem einer für den schulunterricht angemessenen Einführung und Entwicklung des Wahrscheinlichkeitsbegriffs.

\section{Zum Wahrscheinlichkeitsbegriff im Mathematikunterricht und in der
mathematischen Forschung}

Sieht man sich, bezogen auf diese Problematik, einige Mathematikschulbücher an, so lassen sich folgende Tendenzen feststellen: Für die sekundarstufe I, in welcher die Wahrscheinlichkeitsrechnung ein zusätzliches stoffgebiet darstellt und auch in den Büchern vom 5. bis zum 10. Schuljahr nur sporadisch angeboten wird, gilt im großen und ganzen, daß man zunächst mit kombinatorischen Methoden beginnt und dementsprechend anschließend die wahrscheinlichkeit in Form von Gleichwahrscheinlichkeit bzw. als "Verhältnis der günstigen Fälle zu allen möglichen Fällen" einführt. Mit dem Begriff der "relativen Häufigkeit", der gerade den operativ-anwendungsbezogenen Aspekt betont und es überhaupt erst ermöglicht, zufallsexperimente durchzuführen, steht man dann vor dem schwierigen Problem seines Verhältnisses zum wahrscheinlichkeitsbegriff: in verschiedenen Experimenten können ja verschiedene relative Häufigkeiten auftreten, obgleich doch die Wahrscheinlichkeit des beobachteten Ereignisses unveränderlich ist. Diese Lücke zwischen Wahrscheinlichkeit und relativer Häufigkeit wird intuitiv mit Hilfe des sogenannten "empirischen Gesetzes der großen Zahlen" geschlossen. Man sagt: Die relative Häufigkeit stabilisiert sich mit wachsender Anzahl der Versuche gegen die wahrscheinlichkeit. 
In der Sekundarstufe II werden meist zusammenhängende Kurse in Wahrscheinlichkeitstheorie durchgefüht und dementsprechend stellen die Lehrbücher eine mehr oder weniger geschlossene Gesantkonzeption für die Wahrscheinlichkeitsrechnung vor. Insgesamt läßt sich dabei eine orientierung am Aufbau von Hochschultexten feststellen: Was angestrebt wird, ist eine axiomatische Grundlegung des Wahrscheinlichkeitsbegriffs etwa nach Kolmogoroff, die nach den je spezifischen Voraussetzungen etwas eher oder später zu Beginn des Kurses erfolgt. Der Begriff der relativen Häufigkeit dient hierbei einer oft heuristisch-begründenden Hinführung zur Wahrscheinlichkeit und zwar einmal bezogen auf die "Sinnhaftigkeit" der Grundregeln des Wahrscheinlichkeitskalküls. Zum anderen verweist die im empirischen Gesetz der großen Zahlen enthaltene Erfahrungstatsache der stabilisierung der relativen Häufigkeiten intuitiv auf die Eindeutigkeit bzw. die Existenz der Wahrscheinlichkeit. Die mit mathematisch-expliziten Definitionen des Wahrscheinlichkeitsbegriffs verbundenen Schwierigkeiten, wie sie z.B. in der von Misesschen Definition der Wahrscheinlichkeit durch den Limes der relativen Häufigkeiten auftreten, erfordern jedoch, so wird vielfach in den schulbüchern konstatiert, eine axiomatisch-implizite Definition dieses Begriffs. "In der modernen Mathematik ist es üblich, Begriffe, die nicht auf schon bekannte Begriffe zurückgeführt werden können, durch ihre wesentlich erscheinenden Eigenschaften zu umschreiben. Dabei steht im vordergrund nicht die Frage nach dem mathematischen Gegenstand selbst, sondern nach seinen strukturellen Eigenschaften. Ihre Gültigkeit kann mit den Mitteln der Mathematik im allgemeinen nicht bewiesen werden, sondern man nimmt sie als giltig an. Solche grundlegend erscheinenden Eigenschaften bezeichnet man als Axiome" (Heigl/Feuerpfeil, 1976, S. 38).

Heuristischer Bezugspunkt und anschauliche Erklärung des Wahrscheinlichkeitsbegriffs sind also sowohl für die Sekundarstufe I als auch für die sekundarstufe II der vermeintliche "Limes" der konvergierenden relativen Häufigkeiten; diese Auffassung reicht jedoch nicht für eine mathematisch saubere Definition, wie sie später durch die Kolmogoroffschen Axiome gegeben wird. Unverkennbar ist m.E. das Bemühen, relativ schnell zu einer sowohl mathematisch exakten als auch möglichst allgemein-abstrakten Definition 
von Wahrscheinlichkeit zu kommen, um dann ledig aller sorgen der Grundlegung des Begriffs oder möglicher zu erwartender Komplikationen (wie sie sich ja z.T. während der Sekundarstufe I beim Übergang von der kombinatorischen Auffassung zur relativen Häufigkeit ergaben) den Gesamtaufbau der stochastik durchführen zu können. Freudenthal bemerkt zu dieser immer stärker werdenden Axiomatisierungstendenz: "Well, after Cantor and Kolmogorov (not to mention Bourbaki) you should be ashamed to do it otherwise. Any piece of mathematics, and in particular, a suspect one like probability needs a figleaf of set theory, ..." (Freudenthal, 1974 , S. 262).

Nun, für die mathematische Forschung hat es historisch auch die Notwendigkeit einer axiomatisch-impliziten Definition gegeben, eine Notwendigkeit, die von vielfältigen Faktoren herrührte und zwar neben den institutionell-sozialen, vor allem in einer sich in großem Ausmaß durchsetzenden Differenzierung und Arbeitsteilung innerhalb der wissenschaftlichen Forschung selbst begründet war. Darüber hinaus sind gerade auch die internen probleme der klassischen Wahrscheinlichkeitstheorie nicht zu übersehen, welche dann im Rahmen der Grundlagenproblematik der gesamten Mathematik zu Anfang dieses Jahrhunderts auf eine "Lösung" drängten. Chincin beschreibt diese situation für die wahrscheinlichkeitstheorie folgendermaßen: "Zu jener zeit herrschte in allen Handbüchern und Aufsätzen zur Wahrscheinlichkeitstheorie uneingeschränkt das alte, von Laplace ohne jegliche Abänderung übernommene Grundlagensystem; es besteht keinerlei Zweifel daran, daß jedem denkenden Forscher die Ungeeignetheit dieses systems, seine Nichtentsprechung gegenüber jenem Niveau, auf das sich die mathematische Wissenschaft seit Laplace' Zeiten aufgeschwungen hat, ganz offenkundig waren; manchmal kann man sogar einzelne episodische Äußerungen aus diesem Anlaß antreffen, und dennoch sprach jeder Autor am Anfang seiner Abhandlung unverändert von den gleichmöglichen und günstigen Fällen, wobei er übrigens bestrebt war, dieses unerquickliche Thema so schnell wie möglich hinter sich $\mathrm{zu}$ bringen und zum weiteren ruhigen Verlauf der Theorie überzugehen, der keine klippen mehr in sich barg. Die Wahrscheinlichkeitstheorie jener zeit wich instinktiv einer un- 
lagen aus. Diese Situation war, wie das immer ist, durch ihre unzureichende logische vollkommenheit bedingt; doch andererseits wurde diese Situation angesichts der Gesamtheit der mathematischen Wissenschaft, die nachdrücklich an der Revision und Umkonstruktion ihres Fundaments arbeitete, immer unzulässiger" (Chincin, 1961, S. 5/6).

Diese Entwicklung führte schließlich zur Kolmogoroffschen Axiomatik, die dann mehr und mehr zur allgemein anerkannten mathematischen Grundlage des Wahrscheinlichkeitsbegriffs und dem einzigen Ausgängspunkt für den Aufbau der Wahrscheinlichkeitstheorie wurde. Die Schulmathematik kann jedoch m.E. nicht in gleicher weise einen so strikten und ausschließlichen Entwicklungsstandpunkt einnehmen, wie ihn die mathematische Forschung vertritt, indem sie etwa ohne jede weitere Erklärung die Axiome an den Anfang ihrer Arbeit stellt. Die Schule muß in stärkerem Ausmaß auch Begründungsfragen berücksichtigen, sie muß deutlicher etwa die Notwendigkeit und Bedeutung von Axiomatisierung darstellen und sich mit verändernden Auffassungen von der Rolle und Aufgabe der Axiomatik (wie etwa dem Unterschied zwischen aristotelischer und moderner Axiomatik) befassen, bevor sie sich auf einen hoch entwickelten axiomatischen standpunkt des mathematischen Begriffs stellen kann.

Ja, man kann sagen, für die Entwicklung des Wahrscheinlichkeitsbegriffs in der schule sind gerade der zusammenhang von Begründung und Entwicklung bzw. das jeweilige Verhältnis von Definition und Anwendung des Begriffs und die wechselseitigen Veränderungen und Differenzierungen in diesem Verhältnis von besonderer Bedeutung; die jeweilige Begriffsdefinition und die zugehörigen Anwendungen regulieren sich gegenseitig, und die Entwicklung des Begriffs wird durch die Erweiterungen des Anwendungsbereichs vorangetrieben. An dem folgenden, etwas längeren zitat wird m.E. sehr schön die je spezifische Bedeutung des Zusammenhangs von Begründungs- und Entwicklungsaspekten des mathematischen Begriffs deutlich, bezogen auf die unterschiedlichen Funktionen in der mathematischen Forschung einerseits und der Schulmathematik andererseits: "In der Wissenschaft bedeutet 'einen Begriff verstehen" eine Theorie entwickeln und umgekehrt ist die Theorie 
insgesamt logisch begründet, wenn sie als ein - ursprünglicher Begriff verstanden werden kann, der entwickelt, konkretisiert und entfaltet wurde. Diese weitestgehende Entwicklung des Begriffs in Form der Theorie begründet selbst den Ausgangsbegriff, obwohl sie umgekehrt auf dieser Grundlage fußt. Der Ausgangspunkt wird durch das Ende begründet oder anders, der Begriff wird durch seine Entwicklung begründet, nicht durch seine Entstehung.

Für die Schule war dieser Standpunkt in seinen Zuspitzungen nie vertretbar, insbesondere weil schüler keine forschenden Mathematiker sind und damit weder über eine differenzierte Erfahrung im Umgang mit Theorien und theoretischen Begriffen verfügen, noch für die Schulmathematik jene Einlinigkeit der fachlichen Forschung vertretbar ist, die die Entstehungs- und Begründungsfragen ausklammert und sich auf rein funktionale Bedeutungsvorstellungen des Begriffs konzentriert. ... Tatsächlich sind aber nun beide Aspekte - den einen wollen wir Begründungsaspekt und den anderen Anwendungsaspekt oder operativen Aspekt nennen - gleich wesentlich, und zwar in ihrem Zusammenhang. Begründung und Anwendung von Begriffen sind gewissermaBen untrennbar verbunden und das macht die Schwierigkeit eines Konzepts der Begriffsentwicklung aus. Der ProzeBcharakter des Begriffs wird begründet durch ein Ineinanderwirken von Begründen, Zurückführen, Elementarisieren, Interpretieren einerseits und Anwenden, operativ als Werkzeug entwickeln, andererseits. Beide Aspekte verhalten sich gegensätzlich zueinander, d.h. jeder setzt dem anderen die Grenze." (Keitel/Otte, 1977, III-6ff)

Was es so schwer macht, den in diesem zitat aufgefürten besonderen Anforderungen der Schulmathematik nachzukommen und stärker Begründungsfragen $z$ berücksichtigen, ist nun die Tatsache, daß die moderen Axiomatik, die für den Wahrscheinlichkeitsbegriff in gewisser Weise ja "wirklich" erst eine befriedigende Definition darstelit, geradezu Ausdruck des strikten Entwicklungsstandpunktes der mathematischen Forschung ist und daß hier im Grunde sämtliche Begründungsprobleme außer acht gelassen werden.

Aber auch diese Auffassung von Axiomatik hat sich erst historisch entwickelt. Wurden die Axiome in der aristotelischen Methodologie 
als evidente und unbezweifelbare wahrheiten abstrahierter und idealisierter realer Gegenstände aufgefaßt, so sind sie in der Hilbertschen Methodologie weder evidente Wahrheiten noch ist es sinnvoll danach zu fragen, ob sie richtig sind. Die historische Entwicklung der Wahrscheinlichkeitstheorie kann in gewisser Weise (neben der Geometrie) als Beispiel für eine sich verändernde Auffassung von der Aufgabe der Axiomatik verstanden werden. Dabei wird der übergang von einer aristotelischen Methodologie, wie sie sich etwa im Begriff der Gleichwahrscheinlichkeit, bezogen auf den idealen würfel darstellt, (wenn dies auch historisch nie explizit so formuliert wurde) zu einer modernen Axiomatik deutlich, wie Hilbert sie in seinem sechsten Problem gefordert und Kolmogoroff sie schließlich 1933 gegeben hat. Auch Freudenthal betont in seinem Aufsatz: "The Implicit Philosophy of Mathematics today" die hervorragende Rolle der Wahrscheinlichkeitstheorie für die Entwicklung der modernen Axiomatik: "In any case, probability is the field where at present non-Aristotelian methodology is the most clearly understood. This fact, and the tremendous progress of the last 30 years in probability and statistics is disregarded by Aristotelian methodologists, who invent new probability theories, based on explicit definitions of the fundamental notions." (Freudenthal, 1968, S. 352)

Will man auf der schule bei der Entwicklung des Wahrscheinlichkeitsbegriffs ernsthaft der oben gestellten Forderung nach einem angemessenen Verhältnis von Begründungs- und Entwicklungsmomenten des Begriffs nachkommen, so zeigen die gemachten Anmerkungen zum Problem der axiomatischen Definition des Wahrscheinlichkeitsbegriffs, daß man neben der rein mathematischen Entwicklung des Stoffes auch mehr über die Mathematik selbst sagen muB; d.h. es muß verstärkt Wissen über mathematisches Wissen, etwa Wissen über die Rolle von Axiomatik und mathematischer Theoriestruktur vermittelt werden, soll der Schüler nicht nur mathematische Techniken erwerben, sondern auch Sinn und Nützlichkeit dieser Techniken einschätzen und sie für den je spezifischen Gebrauch angemessen variieren können.

Für das hier speziell vorgestellte Problem einer angemessenen Entwicklung und Begründung des Wahrscheinlichkeitsbegriffs heiBt 
dies vor allem, daß der Schüler mehr über die jeweilige Auffassung und besondere Art der Definition des Wahrscheinlichkeitsbegriffs erfährt und sieht, welche Notwendigkeiten es erforderlich machen, den Begriff zu entwickeln und zu erweitern und dabei gleichzeitig eine Veränderung des Charakters der mathematischen Definition dieses Begriffs vorzunehmen.

Am Beispiel des "Gesetzes der großen Zahlen" wollen wir nun versuchen, diesem Problem etwas näherzukommen und das oben erwähnte Verhältnis von Begründung (Definition) und Anwendung des mathematischen Begriffs exemplarisch zu verdeutlichen. Eine Herangehensweise an dieses Theorem also, bei der nicht aufgrund schon entwickelter Begriffe der Satz quasi technisch schnell "erledigt" wird, sondern bei der umgekehrt mit Hilfe des satzes der Wahrscheinlichkeitsbegriff selbst weiterentwickelt wird. Dazu wollen wir ausführlicher die historische situation betrachten, in welcher man ja genau vor dieser problematischen Aufgabe stand: Ohne jegliche allgemeinen Begriffe wie etwa Zufallsvariable, Erwartung, Erwartung einer Zufallsvariablen usw. galt es, den Satz $\mathrm{zu}$ formulieren und anschließend $\mathrm{zu}$ beweisen.

\section{Jakob Bernoulli und sein Theorem}

Jakob Bernoullis Werk uber Wahrscheinlichkeitstheorie "Ars conjectandi" (oder: die Kunst des Mutmaßens) stellt einen entscheidenden konzeptionellen Fortschritt in der frihen phase der Wahrscheinlichkeitstheorie dar, so die einhellige Meinung vieler Mathematiker und Historiker (vgl. etwa Hacking, Todhunter, Kolmogoroff). Das Neue und überaus wichtige war natürlich das heute nach ihm benannte "Bernoullische Theorem", welches im vierten und letzten Teil des Werkes bewiesen wird. Es ist das erste, wichtige Theorem der Wahrscheinlichkeitstheorie uberhaupt.

Mehr als 20 Jahre lang arbeitete Bernoulli an seinem Buch, nie war er richtig zufrieden damit; und erst 8 Jahre nach seinem Tode (1705) veröffentlichte der Neffe Nicholas Bernoulli die "Ars conjectandi" 1713 in Basel. Dies läßt vielleicht etwas von den Schwierigkeiten der inhaltlichen Thematik des Buches insgesamt 
und insbesondere des Theorems selbst erahnen, und es macht deutlich, mit welcher Vorsicht Bernoulli zu Werke ging; zumal hinzugefügt werden muß, daß die "Ars conjectandi" unvollständig blieb, insofern als Bernoulli im AnschluB an den Beweis seines Theorems eigentlich nichts mehr bezüglich des intendierten Vorhabens des 6. Teils sagt, wie es sich in der überschrift ankündigt: "Anwendung der vorhergehenden Lehre auf bürgerliche, sittliche und wirtschaftliche verhältnisse."

Was waren nun die begrifflichen Voraussetzungen der Wahrscheinlichkeitstheorie, auf die Bernoulli sich zu seiner zeit stützen konnte? In welchem konzeptionellen Rahmen hat er sein Theorem formuliert und bewiesen?

Mit dem Briefwechsel zwischen Pascal und Fermat im Jahre 1654 über die beiden vom Chevalier de Méré gestellten Glückspielprobleme wird die Geburt der Wahrscheinlichkeitstheorie angesetzt. Sicherlich erklärt die Tatsache der bloßen Problemformulierung nicht die Herausbildung der Wahrscheinlichkeitstheorie zu diesem Zeitpunkt; es müßte mehr über die notwendigen Voraussetzungen und Veränderungen, wie sie etwa für die Entstehung der neuzeitlichen Wissenschaften insgesamt erforderlich waren, gesagt werden (man muß quasi mehr über "zeugung" und "Schwangerschaft" wissen, will man die "Geburt" verstehen; vgl. zur Vorgeschichte der Wahrscheinlichkeitstheorie u.a. das Buch von Hacking). Im Verlaufe der ersten Phase der Wahrscheinlichkeitstheorie zeigte sich jedoch, welch" zentrale Rolle das "Paradigma" des Glücksspiels für die Entstehung des mathematischen wahrscheinlichkeitsbegriffs spielte. Neben den schon erwähnten Aufgaben des Chevalier de Méré wird dies überaus deutlich sichtbar in dem ersten Büchlein zur Wahrscheinlichkeitstheorie von Christian Huygens: "Ubber das Rechnen in Glücksspielen" (1657, Ratiociniis in aleae ludo), in Jakob Bernoullis "Ars conjectandi", in Rémond de Montmorts "Essai d'analyse sur les jeux de hazaras" $(1708 / 1713)$, in Abraham de Moivres "The Doctrine of chances" (1718) und in den so vielzähligen Problemen zur wahrscheinlichkeitsrechnung, die fast immer in die Form von Glücksspielen eingekleidet wurden. Man wird die Wahrscheinlichkeitstheorie dieser Zeit bestimmt nicht einfach ausschlieBlich als Theorie der Glücks- 
spiele auffassen und erklären können, denn von Anfang an gab es mehr oder weniger heuristische vorstellungen von allgemeiner Nützlichkeit und Anwendbarkeit der Wahrscheinlichkeitstheorie in den verschiedensten Bereichen wie z.B. in den Sterblichkeits- und Rentenberechnungen (vgl. Graunt, 1662 und Hudde/de Witt, 1671). Es sind jedoch die Glücksspiele und darunter vor allem das würfel- und Kartenspiel, die in quasi natürlicher Weise den Erklärungsrahmen für den Wahrscheinlichkeitsbegriff abgeben: innerhalb dieses vorgegebenen Rahmens, der sich auf die idealisierten Gegenstände, würfel oder Kartenspiel bezieht, ist die Bedeutung des Begriffs Wahrscheinlichkeit eigentlich sofort klar; der Unabhängigkeitsbegriff ergibt sich ebenso wie die Gültigkeit von Additions- und Multiplikationstheorem aus den idealen Eigenschaften des Glücksspiels. Kurz, die Vorstellung des idealen Spiels stellt zugleich einen natürlichen Erklärungsrahmen für das grundlegende Begriffs- und Regelsystem der Wahrscheinlichkeitstheorie zur Verfügung und ermöglicht zudem in diesem Rahmen die Formulierungen neuer, die Theorie vorantreibender Probleme.

Dieser frühe Wahrscheinlichkeitsbegriff ist in erster Linie ein kombinatorisch-"deterministischer" Begriff; die Zufälligkeit von Ereignissen spielt gegenüber der idealen symmetrie von Gegenständen noch nicht die wichtige Rolle; zudem ist insofern noch kein Unterschied zwischen Theorie und Anwendung festzustellen, als die Theorie eindeutig durch die idealen Gegenstände erklärt wird.

A.N. Kolmogoroff beschreibt diese erste Phase mit ihrem kombinatorischen Gleichwahrscheinlichkeitsbegriff folgendermaßen: "Es ist die Periode der Entstehung der elementaren Grundbegriffe unserer Wissenschaft, der elementaren Theoreme vom Typ des Additions- und Multiplikationstheorems der Wahrscheinlichkeiten und der elementar-arithmetischen und kombinatorischen Methoden. Als konkretes Material dienen hier vor allem Aufgaben aus dem Bereich der Glücksspiele: Würfelspiele, Kartenspiele und dergleichen. Paradoxerweise kann diese Periode ungeachtet dessen vor allem die philosophische Periode der Entwicklung der Wahrscheinlichkeitstheorie genannt werden." (Kolmogoroff, 1947, S. 4) 
Damit nimmt Kolmogoroff auf die umstrittene erkenntnistheoretische Interpretation des Wahrscheinlichkeitsbegriffs Bezug: Handelt es sich hierbei um einen objektiven, "in der Wirklichkeit vorkommenden" oder um einen subjektiven, vom Menschen willkürlich konstruierten Begriff? Der Philosoph Hacking betont, daß dex Wahrscheinlichkeitsbegriff gleichzeitig mit seiner Entstehung diesen dualen Charakter, diesen Januskopf aleatorischer und epistemologischer Bedeutung hatte (vgl. Hacking, 1975, S. 12). Der erste, der von "objektiver" und "subjektiver" Gewißheit bezogen auf die Wahrscheinlichkeitstheorie spricht, ist Jakob Bernoulli; und auch nur seine Wahrscheinlichkeitsinterpretation wollen wir hier, ohne uns ausführlicher in diese schwierige Diskussion einzulassen, kurz darstellen, um besser die Grundlagen für sein Grenztheorem zu verstehen.

Zu Beginn des 4. Teils der "Ars conjectandi" im ersten Kapitel: "Einleitende Bemerkungen iber Gewißheit, Wahrscheinlichkeit, Notwendigkeit und Zufälligkeit der Dinge" erklärt Bernoulli den Unterschied objektiver und subjektiver Gewißheit: "Die Gewißheit irgendeines Dinges läßt sich entweder objektiv, a.h. an sich betrachten, und bezeichnet in diesem Falle nichts anderes als das wirkliche gegenwärtige oder zukünftige Vorhandensein jenes Dinges, oder subjektiv, d.h. in bezug auf uns und besteht dann in dem Maße unserer Erkenntnis hinsichtlich dieser Wirklichkeit." (Bernoulli, 1899, S. 71) Jedoch "aufgrund göttlicher Voraussicht und Vorherbestimmung" (S. 72), so Bernoulli, hat "alles, was unter der sonne existiert oder entsteht, das Vergangene, das Gegenwärtige und das zukünftige ... an sich die höchste Gewißheit." (S. 71

Bezogen auf uns Menschen "variiert jedoch, so Bernoulli, die betrachtete Gewißheit der Dinge ... vielfach nach oben und unten". Und Bernoulli fährt fort: "Jene Dinge, von welchen es uns durch Offenbarung, Ubberlegung, sinnliche Wahrnehmung, Erfahrung, Autopsie oder irgendwie anderes gewiß ist, daß wir an ihrer gegenwärtigen oder zukünftigen Existenz nicht zweifel haben dürfen, besitzen für uns die höchste und absolute Gewißheit. Alle übrigen Dinge erhalten ein gemäß unserer Erkenntnis unvollkommeneres Maß der GewiBheit, welches größer oder kleiner ist, je nachdem mehr oder weniger wahrscheinlichkeiten dafür vorhanden sind, daß ir- 
gendein Ding ist, sein wird oder gewesen ist." (S. 72)

Damit kann Bernoulli nun die Wahrscheinlichkeit folgendermaßen interpretieren: "Die Wahrscheinlichkeit ist nämlich ein Grad der GewiBheit und unterscheidet sich von ihr wie ein Teil vom Ganzen. iorn z.B. die volle und absolute Gewißheit, welche wir mit a oder 1 bezeichnen, aus fünf Wahrscheinlichkeiten oder Teilen bestehend angenommen wird, von denen drei für das gegenwärtige oder zukünftige Eintreten irgendeines Ereignisses und die übrigen beiden dagegen sprechen, so soll das Ereignis $\frac{3}{5} \cdot$ a oder $\frac{3}{5}$ der Gewißheit besitzen." (S. 72)

Auf der Grundlage seiner allgemeinen Vorstellung von Wahrscheinlichkeit als einem Teil-Ganze-Verhältnis kann Bernoulli in bestimmten Fällen von einem kombinatorischen (Gleich-)Wahrscheinlichkeitsbegriff ausgehen, der sich a priori aus den idealen Eigenschaften (etwa der symmetrie) betrachteter Ereignisse ergibt. Dabei ist dieser Begriff Ausdruck der Unsicherheit und Ungewißheit des unvollkommenen Menschen uber die prinzipiell unterliegende absolute GewiBheit.

Was macht es nun notwendig, diesen doch relativ in sich geschlossenen Begriffskontext zu erweitern und was erfordert es, den Begriff der relativen Häufigkeit, wie er sich ja im Bernoullischen Theorem zeigt, mit dem der Gleichwahrscheinlichkeit in einen mathematischen zusammenhang zu bringen?

Im Kapitel IV: "Über die zwei Arten, die Anzahl der Fälle zu ermitteln. Was von der Art, sie durch Beobachtung zu ermitteln, zu halten ist. Hauptproblem hierbei und anderes." hebt Bernoulli hervor, daß es nur in den wenigsten Fällen möglich ist, die Wahrscheinlichkeit a priori aus den idealen Eigenschaften des betrachteten Gegenstandes zu ermitteln, in den allermeisten Anwendungen der Wahrscheinlichkeitstheorie auf zufällige Ereignisse ist dagegen diese Berechnungsweise undurchführbar. Ich möchte hier eine etwas längere Passage zitieren, in der Bernoullis uberlegungen deutlich zum Ausaruck kommen, und man erkennt, daß der erste Impuls für die Verallgemeinerung und Entwicklung des Gleichwahrscheinlichkeitsbegriffs von praktischen Anforderungen her- 
rührt: Man möchte die Wahrscheinlichkeitstheorie auf eine sehr viel größere Klasse von Gegenständen anwenden können. Zunächst stellt Bernoulli fest: "Wir sind also dahin gelangt, daß zur richtigen Bildung von Vermutungen über irgendeine Sache nichts anderes zu tun erforderlich ist, als aaß wir zuerst die zahl dieser Fälle genau ermitteln und dann bestimmen, um wieviel die einen Fälle leichter als die anderen eintreten können." (S. 88) Damit ist offensichtlich die kombinatorische Gleichwahrscheinlichkeit gemeint. Sofort kommt Bernoulli auf die Problematik dieser Wahrscheinlichkeitsdefinition zu sprechen: "Und hier scheint uns gerade die Schwierigkeit zu liegen, da nur für die wenigsten Erscheinungen und fast nirgends anders als in Glücksspielen dies möglich ist; die Glücksspiele wurden aber von den ursprünglichen Erfindern, damit die Spielteilnehmer gleiche Gewinnaussichten haben sollten, so eingerichtet, daß die zahlen der Fälle, in welchen sich Gewinn oder Verlust ergeben muß, im voraus bestimmt und bekannt sind und daß alle Fälle mit gleicher Leichtigkeit eintreten können. Bei den weitaus meisten anderen Erscheinungen aber, welche von dem walten der Natur oder von der Willkür der Menschen abhängen, ist dies keineswegs der Fall. So sind z.B. bei Würfeln die zahlen der Fälle bekannt, denn es gibt für jeden einzelnen Würfel ebenso viele Fälle wie es Flächen hat; alle diese Fälle sind auch gleich leicht möglich, da wegen der gleichen Gestalt aller Flächen und wegen des gleichmäßig verteilten Gewichts des Würfels kein Grund dafür vorhanden ist, daß eine Würfelfläche leichter als eine andere fallen sollte, was der Fall sein würde, wenn die würfelflächen verschiedene Gestalt besäßen und ein Teil des Würfels aus schwererem Material angefertigt wäre als der andere Teil. ... Welcher sterbliche könnte aber je die Anzahl der Krankheiten (das ist ebensovieler Fälle), welche den menschlichen körper an allen seinen Teilen und in jedem Alter befallen und den Tod herbeiführen können, ermitteln und angeben, um wieviel leichter diese als jene Krankheit, die Pest als die Wassersucht, die Wassersucht als Fieber den Menschen zugrunde richtet, um daraus eine vermutung über das verhältnis von Leben und sterben küntiger Geschlechter abzuleiten? ... Da diese und ähnliche Dinge von ganz verborgenen Ursachen abhängen, welche überdies noch durch die unendliche Mannigfaltigkeit ihres $\mathrm{Zu}-$ sammenwirkens unsere Erkenntnis beständig täuschen, so würde es 
sinnlos sein, auf diese Weise etwas erforschen zu wollen." (S. 88/89) Wie aber kann man sonst in diesen Fällen die wahrscheinlichkeit bestimmen? Bernoulli fährt fort: "Aber ein anderer Weg steht uns hier offen, um das Gesuchte zu finden und das, was wir a priori nicht bestimmen können, wenigstens a posteriori, d.h. aus dem Erfolge, welcher bei ähnlichen Beispielen in zahlreichen Fällen beobachtet wurde, zu ermitteln." (S. 89)

Man soll also mit Hilfe der beobachteten relativen Häufigkeit die Wahrscheinlichkeit berechnen. "Diese empirische Art, die Zahl der Fälle durch Beobachtungen zu bestimmen, ist weder neu noch ungewöhnlich, ... und alle Menschen beobachten im täglichen Leben dasselbe Verfahren. Auch leuchtet jedem Menschen ein, daß es nicht genügt, nur eine oder die andere Beobachtung anzustellen, um auf diese Weise über irgendein Ereignis zu urteilen, sondern daß eine große Anzahl von Beobachtungen erforderlich sind. Zuweilen hat auch schon ein recht einfältiger Mensch infolge irgendeines natürlichen Instinktes von sich aus und ohne jede vorangegangene Unterweisung die Erfahrung gemacht (was wirklich wunderbar ist), daß man, je mehr diesbezügliche Beobachtungen vorliegen, umso weniger Gefahr läuft, von der wahrheit abzuirren." Man muB jedoch achtgeben; dieses, heute würde man sagen empirische Gesetz der großen Zahlen, bedarf nämlich einer genauen Analyse. Bernoulli merkt kritisch an: "Obgleich nun dies aus der Natur der sache heraus von jedem eingesehen wird, so liegt doch der auf wissenschaftlichen Prinzipien gegründete Beweis durchaus nicht auf der Hand, und es liegt mir daher ob, ihn an dieser stelle zu erbringen." (S. 90) Darüber hinaus ist es für Bernoulli wichtig festzustellen, ob man sich nicht mit Hilfe der Methode der relativen Häufigkeiten vielleicht nur asymptotisch einem bestimmten Grad der GewiBheit annähern kann, welcher etwa nur einen Bruchteil der absoluten Gewißheit darstellt; zudem mu der Zusammenhang zwischen Anzahl der benötigten Beobachtungen und der Genauigkeit der Annäherung an die Wahrscheinlichkeit untersucht werden.

Aus diesen hier ausführlich dargestellten Vorüber legungen Bernoullis wird zweierlei ersichtlich. Zum einen ergibt sich aus der Erweiterung des Anwendungsbereichs von den idealsymmetrischen 
Gegenständen zu den zufälligen Ereignissen die Notwendigkeit, einen zusammenhang zwischen dem bisherigen Begriff der Gleichwahrscheinlichkeit und dem der relativen Häufigkeit herzustellen, was m.E. zu einer Differenzierung zwischen dem theoretischen Begriff einerseits und den praktischen Anwendungen andererseits führt und letztendlich auf eine Erweiterung des Wahrscheinlichkeitsbegriffs selbst hinausläuft. Zum zweiten erkennt man deutlich die Anforderungen mathematischer strenge, die Bernoulli an diesen $\mathrm{Zu-}$ sammenhang stellt, und man wird diesen "Vorsichtsmaßnahmen" nur beipflichten können, kennt man die doch recht komplizierte mathematische Struktur des Bernoullischen Theorems, welches dann auch, so das Urteil Kolmogoroffs mit "vollkommener analytischer strenge" bewiesen wurde (vgl. Maistrov, 1974, S. 75).

Im V. und letzten Kapitel wird dieser Beweis durchgeführt. In Bernoullis Sprechweise lautet der Satz: "Es möge sich die zahl der günstigen Fälle zu der $Z$ ahl der ungüstigen Fälle genau oder näherungsweise wie $\frac{r}{s}$, also zu der zahl aller Fälle wie $\frac{r}{r+s}=\frac{r}{t}-$ wenn $r+s=t$ gesetzt wird - , verhalten, welches letztere verhältnis zwischen den Grenzen $\frac{r+1}{t}$ und $\frac{r-1}{t}$ enthalten ist. Nun können, wie zu beweisen ist, so viele Beobachtungen gemacht werden, daß es beliebig oft (z.B. c-mal) wahrscheinlicher wird, daß das Verhältnis der günstigen zu allen angestellten Beobachtungen innerhalb dieser Grenzen liegt als außerhalb derselben, also weder größer als $\frac{r+1}{t}$ noch kleiner als $\frac{r-1}{t}$ ist." (S. 104)

Diese Formulierung bringt deutlich die Einbeziehung der zufälligkeit in den Wahrscheinlichkeitskalkül zum Ausdruck.

Mathematisch formaler läßt sich Bernoullis Theorem folgendermaßen übersetzen: "Sei die Wahrscheinlichkeit des betrachteten Ereignisses $p=\frac{r}{t}$ und seien die Genauigkeitsgrenzen $\frac{\mathrm{kr} \pm 1}{\mathrm{kt}}$; dann können, wie $z u$ beweisen ist, so viel Beobachtungen gemacht werden, daß gilt:

$$
P\left(h_{n} \varepsilon\left[\frac{k r-1}{k t}, \frac{k r+1}{k t}\right]\right) \longrightarrow 1
$$

(bzw. $P>\frac{c-1}{c}$ für jedes beliebige $c$ ), wobei $h_{n}$ die beobachtete relative Häufigkeit ist." 
Wir haben hier die Exaktheitskonstante $\mathrm{k}$ explizit eingeführt, während Bernoulli, wie es aus seiner Darstellung deutlich wird, implizit immer noch von einem "der jeweiligen situation genügend feinen" Verhältnis $\frac{r}{t}=\frac{k r}{k t}$ ausgeht.

Die geniale Beweisidee Bernoullis beruht darauf, die Binomialentwicklung von $(r+s)^{n(r+s)}$ zu studieren, das größte Glied hiervon zu bestimmen und die summe der $2 n+1$ Glieder zu berechnen, die sich neben dem größten aus den je $n-G l i e d e r n$ links und rechts davon ergibt. Dann kann, wie im Satz behauptet, nachgewiesen werden, daß "so viele Beobachtungen angestellt werden (können), daß die Anzahl der Fälle, in welchen das verhältnis der günstigen zu allen überhaupt angestellten Beobachtungen die Grenzwerte $\frac{n \cdot r+n}{n \cdot t}$ and $\frac{n \cdot r-n}{n \cdot t}$ oder $\frac{r+1}{t}$ und $\frac{r-1}{t}$ nicht überschreitet, mehr als c-mal größer ist als die summe der übrigen Fälle, d.h., daß es mehr als c-mal wahrscheinlicher wird, daß das Verhältnis der Anzahl der günstigen zu der Anzahl aller Beobachtungen die Grenzen $\frac{r+1}{t}$ und $\frac{x-1}{t}$ nicht überschreitet, als daB es sie überschreitet." (S. 105/106)

In moderner Schreibweise wird dem Bernoullischen Theorem oft die Form gegeben:

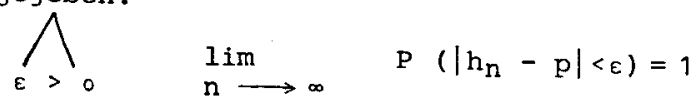

Hierin drückt sich ausschließlich die Tatsache aus, daB letztlich mit Sicherheit die beobach tete relative Häufigkeit mit der wahrscheinlichkeit des Ereignisses genau übereinstimmt; die Häufigkeiten "stabilisieren" sich also beliebig genau um die wahrscheinlichkeit. Es war tatsächlich auch das Hauptinteresses Bernoullis nachzuweisen, daß mit beliebiger Gewißheit wahrscheinlichkeit und relative Häufigkeit sich beliebig einander annähern, wenn nur die Anzahl der Beobachtungen groß genug gemacht wird.

Man kann dieses hier in seinem Aspekt der bloBen Konvergenz vorgestellte Theorem jedoch auch anders interpretieren, wenn nach "Schnelligkeit" der Konvergenz bzw. nach einer groben Abschätzung dieser schnelligkeit gefragt wird, was dann deutlicher die hier anzutreffende Art und Weise der konvergenz hervorhebt und die 
mathematische Struktur des Bernoullischen Satzes und die darin steckenden technischen und begrifflichen Probleme deutlich werden läßt. In moderner Notation hätte man hierfür etwa die folgende Form, deren strukturelle Ähnlichkeit mit dem "übersetzten" Bernoulli-Theorem sofort auffällt:
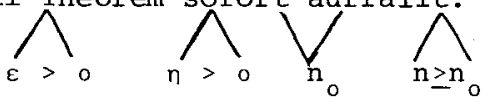

$$
P\left(\left|h_{n}-p\right|<\varepsilon\right)>1-n
$$

Insgesamt hat man also drei variable Größen: einmal die Exaktheit der betrachteten Aussage, welche durch $\varepsilon$ gemessen wird, dann die Sicherheit, mit welcher die Aussage gilt, gemessen durch $n$ und schließlich die Anzahl der gemachten Versuche, gegeben durch $n$. Diese drei Parameter bedingen sich gegenseitig, man kann jeweils zwei festlegen und dann versuchen, den dritten abzuschätzen.

Es ist nun interessant zu sehen, daß auch Bernoul1i, obwohl eigentlich ausschließlich an der Konvergenz interessiert, sich diesem Problem widmet. Hatte er die Konvergenzaussage zunächst mit Hilfe von Unendlichkeitsargumenten auch hergeleitet, so gibt er einen zweiten Beweis, da "von denen, die sich nicht mit unendlichkeitsbetrachtungen befreundet haben" (S. 100) Einwände gemacht werden könnten. "Diesen Bedenken kann ich nicht besser entgegentreten, als daß ich jetzt die Berechnung für einen endlichen Wert von $n$ wirklich durchführe ..." (s. 100), so Bernoulli. Diese Rechnung ergibt dann die folgende von Karl Pearson wohl zu Recht als sehr grob kritisierte Abschätzung für den Zusammenhang der drei Größen $\varepsilon, n$ und $n$.

Bezeichnet man mit $r$ die günstigen, mit $s$ die ungünstigen und mit $t=r+s$ alle möglichen Fälle, und sei die Exaktheit durch das Intervali

$$
\left[\frac{k r-1}{k t}, \frac{k r+1}{k t}\right]=\left[\frac{r^{\prime}-1}{t^{\prime}}, \frac{r^{\prime}+1}{t^{\prime}}\right]
$$

gegeben und werde mit $c$ die Sicherheit der Aussage angegeben (und zwar in der Form, daB es c-mal wahrscheinlicher ist, die relative Häufigkeit innerhalb des obigen Intervalls als außerhalb desselben zu finden), so wähle man für die Anzahl der durchzufihrenden Versuche den größeren der beiden folgenden werte: 
$n_{1} t^{\prime}=m t^{\prime}+\frac{s^{\prime} t^{\prime}(m-1)}{r^{\prime}+1}$, wobei $m \geq \frac{\log c\left(s^{\prime}-1\right)}{\log \left(r^{\prime}+1\right)-\log r^{\prime}}$

und

$n_{2} t^{\prime}=m t^{\prime}+\frac{r^{\prime} t^{\prime}(m-1)}{s^{\prime}+1}$, wobei $m \geq \frac{\log c\left(r^{\prime}-1\right)}{\log \left(s^{\prime}+1\right)-\log s^{\prime}}$

Mit Hilfe dieser komplizierten Formeln lassen sich nun tatsächlich aus je zwei vorgegebenen Größen grobe Abschätzungen für die restlichen ermitteln. Bernoulli kommt etwa in einem von ihm durchgerechneten Beispiel zu dem Resultat, daß bei einem Ereignis mit Wahrscheinlichkeit $\frac{3}{5}$ "nach dem oben bewiesenen Satz(es) mehr als 1000-mal wahrscheinlicher ist, daß bei 25550 angestellten Beobachtungen das Verhältnis der günstigen zu allen Beobachtungen innerhalb der Grenzen $\frac{31}{50}$ und $\frac{29}{50}$ (diese einschließlich) liegt als außerhalb derselben." (S. 107)

Bernoulli war in erster Linie an einer zurückführung der relativen Häufigkeit auf die Gleichwahrscheinlichkeit interessiert, so wie er es auch in den letzten Sätzen seines Werkes betont: "Wenn also alle Ereignisse durch alle Ewigkeit hindurch fortgesetzt beobachtet würden (wodurch schließlich die Wahrscheinlichkeit in volle GewiBheit übergehen müßte), so würde man finden, daß alles in der Welt aus bestimmten Gründen und in bestimmter GesetzmäBigkeit eintritt, daB wir also gezwungen werden, auch bei noch so zufällig erscheinenden Dingen eine gewisse Notwendigkeit, und sozusagen ein Fatum anzunehmen." (S. 107)

In dem von Bernoulli zusätzlich durchgeführten "endlichen" Beweis jenes Satzes zeigt sich jedoch im Keim ein grundsätzliches strukturelles Problem des Wahrscheinlichkeitsbegriffs. Geht man von ideal-geometrischen Gegenständen auf zufällige Ereignisse über, so läßt sich die Wahrscheinlichkeit nun nicht mehr a priori aus der Symmetrie in quasi "deterministischer" Weise bestimmen, die Wahrscheinlichkeit (als relative Häufigkeit) eines zufälligen Ereignisses wird quasi selbst zu einer zufallsgröße. Dies wird aus dem komplizierten zusammenhang zwischen relativer Häufigkeit und Wahrscheinlichkeit, den Bernoullis Theorem gerade zum Ausdruck bringt, deutlich: Die relative Häufigkeit ist ein "statistisches" 
Maß für die Wahrscheinlichkeit.

Im Grunde wird im Bernoulli-Theorem ein "Zirkel" sichtbar, der darauf verweist, daß man eigentlich immer schon vorher wissen muß, was Wahrscheinlichkeit "ist", will man die Wahrscheinlichkeit eines bestimmten zufälligen Ereignisses durch die beobachtete relative Häufigkeit erklären. Denn dieser Erklärungszusammenhang unterliegt wiederum selbst einer Wahrscheinlichkeit, die man somit voraussetzen muß.

Man könnte sagen, Bernoulli versuche sich den Schwierigkeiten dieser Zirkularität dadurch zu entziehen, daß er zum idealen Grenzfall ibergeht; bei unendlich vielen versuchen erhält man scheinbar eine einfache Definitionsgleichung $h_{n}=p$. Jedoch auch hier wird, wie es sich im starken Gesetz der großen Zahlen zeigt: $P\left(\lim _{n \rightarrow \infty} h_{n}=\frac{r}{t}\right)=1$, wiederum von wahrscheinlichkeit Gebrauch gemacht. In der statistischen praxis, in der man es immer mit einer endlichen Versuchszahl zu tun hat, benutzt man das Konzept der "kleinen Wahrscheinlichkeiten". Man vernachlässigt kleine Unsicherheiten und nimmt die entsprechende relative Häufigkeit als die Wahrscheinlichkeit. Hierbei muß man jedoch vorher zumindest schon wissen, was kleine bzw. große wahrscheinlichkeiten sind. Die hier angesprochene Zirkularität des Wahrscheinlichkeitsbegriffs wird in den verschiedensten Formen sichtbar. So steckt letztlich bereits in der Annahme der Gleichwahrscheinlichkeit, von einem entwickelteren standpunkt her gesehen, dieser Aspekt; auch hier wird wahrscheinlichkeit schon vorausgesetzt. Desweiteren hängt die in Bernoullis Theorem vorkommende "Sicherheits"Wahrscheinlichkeit $P$ von der Wahrscheinlichkeit $\frac{r}{t}$ des Ereignisses $\mathrm{ab}$.

All' diese verschiedenen Aspekte der Zirkularität in den Definitionen und Anwendungen des Wahrscheinlichkeitsbegriffs, insbesondere erstmals in Bernoullis Theorem, weisen auf den theoretischen Charakter dieses Begriffs hin: Er kann nicht auf andere reduziert werden, sein Inhalt erschöpft sich nicht in Eigenschaften oder strukturen der von ihm bearbeiteten Gegenstände. Der Zirkel bringt die relative Eigenständigkeit des Begriffs zum Ausdruck. Daneben mu $B$ man jedoch auch die Bedeutung der sich ver- 
ändernden und komplizierter werdenden Anwendungsprobleme für die Entwicklung und Entfaltung des Wahrscheinlichkeitsbegriffs beachten. Um diesen komplizierten Zusammenhang zwischen dem Begriff und seinen Anwendungen richtig zu verstehen, hat es in der Geschichte der Wahrscheinlichkeitstheorie noch mehr als zweihundert Jahre gedauert, und es bedurfte der weiterentwicklung dieses problematischen Zusammenhangs, wie er erstmalig im Bernoullischen Theorem bearbeitet wurde, etwa durch den zentralen Grenzwertsatz über de Moivre und Laplace bis zur Petersburger wahrscheinlichkeitstheoretischen schule, und auBerdem bedurfte es vieler vergeblicher Versuche der expliziten Definition von Wahrscheinlichkeit bis hin zum "frequentistischen Wahrscheinlichkeitsbegriff" von Richard von Mises.

Die jedoch im Bernoulli-Theorem keimhaft angelegte "zirkularität" wird Anfang unseres Jahrhunderts durch Markov, der mehrere Beweise des Bernoullischen Satzes durchführt, anhand seiner Kritik an der bloßen Hervorhebung der Konvergenz der relativen Häufigkeit gegen die Wahrscheinlichkeit deutlich (vgl. Maistrov, 1974, S. 219). Sinngemäß sagt Markov, daß man auch đie scheinbar absolut unwahrscheinlichen Ereignisse berücksichtigen muB, d.h. also sich deutlicher den zufallscharakter der Ereignisse klarmachen muß, was auf die prinzipiell stochastische Natur des Wahrscheinlichkeitsbegriffs hinweist.

Abschließend sei zu Bernoulli angemerkt, daß wix uns bewußt nur auf das hier vorgestellte Problem des quasi zirkelhaften Zusammenhangs zwischen (bekannter) Wahrscheinlichkeit und relativer Häufigkeit konzentriert haben und viele weitere interessante Fragen außer acht haben lassen müssen, wie etwa die nach der Bedeutung des Bernoulli-Theorems für die Statistik oder das Problem der A posteriori-Ermittlung einer "wirklich" unbekannten wahrscheinlichkeit durch die Beobachtung von Ereignissen, wie es etwa Bayes rund 50 Jahre später versuchte. Es sei jedoch nochmals auf die außergewöhnliche Bedeutung des Bernoulli-Theorems hingewiesen, die sowohl in seiner komplizierten theoretischen Struktur als auch in den damit gegebenen größeren Anwendungsmöglichkeiten der Wahrscheinlichkeitstheorie liegt. Die theoretischen Schwierigkeiten, wie sie sich vor allem in der ange- 
sprochenen Zirkularität und dem statistischen Charakter des Wahrscheinlichkeitsbegriffs zeigen, waren wohl vornehmlich die Gründe dafür, warum Bernoulli so lange an seinem Theorem gearbeitet hat. Bezugnehmend auf den statistischen Charakter der Wahrscheinlichkeit - Bernoulli spricht von einer "Annäherung", die zwischen zwei Grenzen eingeschlossen wird (vgl. S. 92) - betont auch Bernoulli selbst über die theoretischen Schwierigkeiten hinaus die Wichtigkeit seines Theorems für die breite Anwendung der Wahrscheinlichkeitsrechnung: "Dieses ist das problem, welches ich an dieser stelle zu veröffentlichen mir vorgenommen habe, nachdem ich schon seit 20 Jahren dasselbe mit mir herumgetragen habe; seine Neuheit sowohl als auch sein außerordentlich großer Nutzen in Verbindung mit seiner ebenso großen Schwierigkeit läßt alle übrigen Kapitel dieser Lehre an Wichtigkeit und Bedeutung gewinnen." (S. 92)

Noch klarer wird die wichtige Rolle, die dieser Satz für die fruchtbare Weiterentwicklung der Wahrscheinlichkeitstheorie spielte aus der Tatsache, daß etwa auch Rémond de Montmort mit seinem Werk ein ähnliches Vorhaben wie Bernoulli versuchte, aber längst nicht diesen Erfolg in der Anwendung seiner Theorie wie Bernoulli erlangte, da es ihm gerade an einem ähnlichen Gesetz der großen $\mathrm{zahl}$ mangelte, welches die Entwicklung des Wahrscheinlichkeitsbegriffs und seine Anwendung auf eine viel breitere Klasse von Gegenständen verhinderte (vgl. dazu Hacking, 1975, S. 145).

\section{Komplementäre Aspekte des Wahrscheinlichkeitsbegriffs - didaktische Anmerkung}

"The history of probability ... shows a stimulating interplay of theory and applications; theoretical progress opens new fields of application, and in turn applications lead to new problems and fruitful research" (Feller, 1968, S. 3). Diese relativ allqemeine Bemerkung Fellers zur sich wechselseitig vorantreibenden Beziehung zwischen Theorie und Praxis der Wahrscheinlichkeitsrechnung wird durch die im vorigen Abschnitt beispielhaft dargestellte Entwicklung des Bernoulli-Theorems bestätigt. Die ange- 
strebte Erweiterung der Anwendungsmöglichkeiten führten zunächst auf der Grundlage des Gleichwahrscheinlichkeitsbegriffs ansatzweise zu einer potentiellen Verallgemeinerung des Wahrscheinlichkeitsbegriffs insgesamt. Bei dem Versuch jedoch, in diesen $\mathrm{Zu}-$ sammenhang die Wahrscheinlichkeit mit Hilfe der relativen Häufigkeit zu messen, zeigte sich eine "Zirkularität": Der MeBvorgang setzt schon den Wahrscheinlichkeitsbegriff voraus, die Art der Messung ist selbst stochastischer Natur. Man kann sagen, daß die Wahrscheinlichkeit nicht mehr aufgrund der symmetrisch-idealen Eigenschaften des Gegenstandes (wie es in der aristotelischen Methodologie üblich ist) von vornherein durch quasi "absolute" Zahlen ausgedrückt werden kann. Der Meßvorgang, d.h. die zuordnung von Zahlwerten zu verschiedenen Wahrscheinlichkeiten, ist viel komplizierter geworden, insofern er nicht theorieunabhängig wie bisher am Anfang steht, sondern in großem Ausmaß die Wahrscheinlichkeitstheorie selbst voraussetzt. Die Weiterentwicklung des Wahrscheinlichkeitsbegriffs wurde, wie schon oben angemerkt, durch die ständigen Erweiterungen des Anwendungsbereichs vom Glücksspiel über zufällige Ereignisse bis hin zu Systemen zufälliger Ereignisse und zu so komplizierten Gegenständen wie z.B. einem Gassystem entscheidend vorangetrieben: Innerhalb dieser sich gegenseitig stützenden Entwicklung von Wahrscheinlichkeitsbegriff und Anwendungsproblemen wurde die theoretische Eigenständigkeit dieses Begriffs relativ $\mathrm{zu}$ seinen Anwendungsgegenständen immer deutlicher. Die Unmöglichkeit der empirischen Grundlegung des Wahrscheinlichkeitsbegriffs wurde besonders eklatant in den Zirkularitäten expliziter Definitionsversuche. Dieser Problematik hat schließlich die Kolmogoroffsche axiomatisch-implizite Begründung dieses Begriffs voll Rechnung getragen. Die moderne Axiomatik macht in gewisser weise ernst mit der Notwendigkeit der theorieabhängigen Anwendung des Begriffs: Es wird nicht mehr am Anfang der Theorie explizit gesagt, was Wahrscheinlichkeit ist, die implizite Definition wird zum Ausdruck der theoretischen Eigenständigkeit des Wahrscheinlichkeitsbegriffs, d.h. sie ist notwendiges Ergebnis der vielfältigen fehlgeschlagenen Bemühungen einer rein reduktionistischen Erklärung von wahrscheinlichkeit.

Nun trifft zwar für die inzwischen "reine" Wahrscheinlichkeits- 
theorie etwa die Bemerkung Doobs zu: "Probability as a purely mathematical subject is not controversial. It is a part of measure theory with specialized terminology ..." (Doob, 1976, s. 197), jedoch wird man auch weiterhin nicht die Bedeutung von Wahrscheinlichkeit ohne ihren Anwendungsbezug verstehen können. Und da dieser Anwendungsbezug insgesamt komplizierter und problematischer geworden ist, so verändert er entscheidend die alte aristotelisch-idealisierte Interpretation von wahrscheinlichkeit. Freudenthal beurteilt diese Situation folgendermaßen: "Kolmogorov's axioms simply state that probability is a measure and that independence is defined by the validity of the multiplication rule. Of course this is not the whole story. The old questions eliminated by Kolmogorov's axiomatics, have not died out. They come back, not in their ontological form, but as questions as to how to apply probability. Not: what is probability? what is independence? but: how can we measure probability? how can we check independence? Tools to answer such questions, and many more, have been mathematized in mathematical statistics." Und zur besonderen Art der wahrscheinlichkeits theoretischen Anwendung sagt Freudenthal weiter unten: "It is a characteristic feature of probability theory that all its applied statements are again made up to a probabilistic error, which is of course numerically specified in every particular case. In the past this has been the occasion of longwinded discussions.", und damit stößt man, wie auch Freudenthal anmerkt, wieder auf den altbekannten zirkel: "... one is still mistrusted by people who would prefer explicit definitions of probability to measuring procedures with a probabilistic error. They consider this to be a circularity. It is a circularity, indeed, yet not a vicious one, but the inherent circularity, of the implicit approach." (Freudenthal, 1968, S. 352)

Hieraus wird also insgesant deutlich: Während der "alte" kombinatorische wahrscheinlichkeitsbegriff a priori durch idealisierte Eigenschaften absolut erklärt wird, so ist für den "neuen" Begriff der stochastische charakter seiner Erklärung zentral.

Innerhalb der Entwicklung der wahrscheinlichkeits theoretischen Methoden zeigt sich diese veränderte sicht auf den Begriff deut- 
lich am Utbergang von Wahrscheinlichkeit als einem zahlverhältnis (kleiner 1) zu Wahrscheinlichkeit als dem Erwartungswert einer Zufallsvariablen (Begriffen also, wie sie sich gerade im Zusammenhang des Gesetzes der großen Zahlen bzw. des zentralen Grenzwertsatzes herausgebildet haben). Im Prinzip ist nun der Erwartungswert einer Zufallsvariablen nicht einfach nur eine Zah1; vielmehr gehört gerade die Verteilung, also die stochastik (wie Freudenthal es nennt, vgl. Freudenthal, 1974, S. 267) immer mit dazu. Und Dinges, der immer wieder die Forderung an den Unterricht in wahrscheinlichkeitstheorie stellt, so etwas wie "stochastisches Denken" dem Schiiler zu vermitteln, einer Denkweise, so Dinges, die es in einem ähnlichen sinne gibt, wie geometrische Vorstellungen, die sich jedoch nicht einfach auf Denkweisen der reinen Mathematik reduzieren lassen, betont in diesem zusammenhang ausdrücklich, daß die schüler in jedem Falle lernen sollten, "daB zu jeder statistischen Aussage eine Irrtumswahrscheinlichkeit gehört" (vgl. Dinges, 1977, S. 3); er hebt also auch den "zufälligen" Charakter der Wahrscheinlichkeit hervor.

Bezogen auf eine praktische Messung, auf einen Versuch also, der nur endlich viele Male wiederholt werden kann, wird dieser zufällige Charakter, ja man könnte sagen, das "statistische Schwanken" der wahrscheinlichkeit besonders schön in der folgenden Komplementarität deutlich sichtbar: Erinnern wir uns zunächst an die drei Variablen des Bernoulli-Theorems, nämlich die Exaktheit $\varepsilon$, die sicherheit $n$ und die Versuchsanzahl $n$. Ist nun $n$, wie es praktisch ja immer nur sinnvoll ist, fixiert, so erkennt man, daß "Exaktheit" und "Sicherheit" sich gegenseitig begrenzen: je exakter man die Aussage im Bernoulli-Theorem z.B. machen will, umso unsicherer wird sie und umgekehrt. Diese Komplementarität bringt anschaulich sehr klar das stochastische verhalten der Wahrscheinlichkeit zum Ausdruck und hebt im Vergleich zum klassischen Begriff die neuen und eigenartigen Charakteristika des veränderten, theoretischen Wahrscheinlichkeitsbegriffs hervor.

Die gemachten Ausführungen haben insgesamt gezeigt, daß man in den Vermittlungsprozessen des Mathematikunterrichts, vor den sich scheinbar gegenseitig widersprechenden problemen des einerseits angemessenen Verhältnisses von Begründungs- und Entwicklungsfragen 
des mathematischen Wissens und andererseits der Theoretizität des mathematischen Begriffs steht. Denn Theoretizität des Begriffs heiBt, daß der Begriff sich letztendlich nur selbst erklären kann, während das Begründungs- und Entwicklungsproblem einen fortdauernden Erklärungsbezug des mathematischen Wissens im Schulunterricht fordert.

Diese Widersprüchlichkeit läßt sich nun m.E. bezogen auf den Wahrscheinlichkeitsbegriff "lösen" und dies sollte auch die historische Entwicklung des Bernoulli-Theorems deutlich gemacht haben, wenn man bewußter den dynamischen Entwicklungscharakter dieses Begriffs hervorhebt. Eine Möglichkeit zur Entwicklung des Wahrscheinlichkeitsbegriffs, welche für eine konkrete unsetzung im Mathematikunterricht jedoch weiterer úberlegungen und Untersuchungen bedarf, besteht gerade darin, gezielt das jeweilige Verhältnis von Begriffsdefinition und Anwendungen und seine Differenzierungen im Auge $z u$ behalten. D.h. man könnte etwa durchaus mit einem kombinatorisch begründbaren Wahrscheinlichkeitsbegriff der zudem zur Herausbildung eines entwickelteren Methodenapparates dient, beginnen. Dabei sollte man jedoch auch schon deutlicher die Entwicklung mit ihren Veränderungen berücksichtigen. Um den notwendigen übergang von der kombinatorischen zur statistischen Wahrscheinlichkeitsauffassung zu erleichtern und für diesen schwierigen theoretischen "Bruch" die erforderlichen mathematischen Methoden und Begriffe bereitzustellen, bietet sich in gewisser Weise die Erarbeitung eines intuitiven Verständnisses der Wahrscheinlichkeitsverteilung durch die deskriptive statistik an; die Wahrscheinlichkeitsverteilung repräsentiert nämlich in einheitlicher Weise die Komplementarität von sicherheit und Exaktheit.

Was insgesamt deutlich werden sollte, ist der notwendigerweise theoretische charakter des Wahrscheinlichkeitsbegriffs, wie er sich in der angesprochenen Zirkularität zeigt; diesem Problem der Theoretizität des mathematischen Wissens kann man nur gerecht werden, wenn man den ProzeBcharakter des Wissens betont. Mit anderen Worten: Der zirkel läßt sich nur in einer permanenten Entwicklung des Begriffs, gesteuert durch die Beziehung zwischen Definition und Anwendungen des Begriffs, "auflösen". 


\section{Literatur}

Bernoulli, Jakob: Wahrscheinlichkeitsrechnung (Ars conjectandi) Ostwald's Klassiker Nr. $107 \mathrm{u} .108$, Leipzig, 1899

Chincin, A.Ja.: Die Häufigkeitstheorie R. von Mises' und die modernen Ideen der Wahrscheinlichkeitstheorie (geschrieben in den Jahren 1939-1944 mit Anmerkungen von B.V. Gnedenkov). In: Voprosy filosofii, 1 (15), 1961, S. 92-102 und 2 (15), 1961 , S. 77-89 (russisch)

de Moivre, Abraham: The Doctrine of Chances or a Method of Calculating the Probability of Events in Play, 1756 , reprinted, Chelsea, New York, 1967 (darin: A Treatise of Annuities on Lives)

Dinges, Hermann: Zum Ungang mit Aussagen in der Stochastik, Mskrpt., 1977

Dinges, Hermann: Schwierigkeiten mit der Bayesschen Regel. In: Math.Phys. Sem., Bd. XXV/1978, Heft 1, S. 113-156

Doob, J.L.: Foundations of Probability Theory and its Influence on the Theory of Statistics. In: Owen, D.B. (ed.): On the History of Statistics and Probability. Marcel Dekker, N.Y., 1976, S. 197-204

Feller, William: An Introduction to Probability Theory and its Applications, vol.1, Wiley, N.Y., 1968

Freudenthal, Hans: The Implicit Philosophy of Mathematics today. In: Klibansky, R. (ed.): Contemporary Philosophy, I Logic and Foundations of Mathematics. Firenze, 1968, S. 342-368

Freudenthal, Hans: The Crux of Course Design in Probability. In: Educational Studies in Mathematics 5 (1974), S. 261-277

Graunt, John: Natural and Political observations mentioned in a following Index, and made upon the Bills of Mortality, London,

Hacking, Ian: The Emergence of Probability. Cambridge University Press, Cambridge, 1975

Heigl, F./Feuerpfeil, J.: Stochastik (Leistungskurs), Bayerischer Schulbuch-Verlag, München, 1976

Huygens, Christian: Ratiociniis in aleae ludo. In: Exercitionum Mathematicorum, ed. F. van Schouten, Amsterdam, 1657

Keitel, Ch./Otte, M.: Das Lehrbuchproblem als Gegenstand der Lehrerausbildung. EPAS-Drucksache Nr. 1, IDM Bielefeld 1977

Kendall, M.G.: On the Reconciliation of Thecries of Probability. In: Biometrika, Jg. $36,1949, \mathrm{~S} .101-116$ 
Kolmogoroff, A.N.: Die Rolle der russischen Wissenschaft in der Entwicklung der Wahrscheinlichkeitstheorie. In: Ukenye Zapiski, Jg. 91, Moskau 1947, S. 53-64 (russisch)

Maistrov, L.E.: Probability Theory, A historical Sketch, Academic Press, N.Y., 1974

Montmort, Pierre Rémond de: Essay d'analyse sur les jeux de hazards, Paris, 1713

Todhunter, I.: A History of the Mathematical Theory of Probability from the Time of Pascal to that of Laplace. Chelsea Pub. Company, N.Y., 1965 (erste Ausgabe 1865)

Heinz Steinbring

Institut für Didaktik der Mathematik

Universität Bielefeld

Postfach 8640

4800 Bielefeld 1 